\title{
Belgeo
}

Revue belge de géographie

$1 \mid 2007$

The advanced service sectors in European urban regions

\section{Le positionnement des villes belges dans le réseau global des services avancés}

The positioning of Belgian cities in the global advanced services network

\section{Laurent Aujean, Etienne Castiau, Marcel Roelandts et Christian Vandermotten}

\section{(2) OpenEdition}

\section{Journals}

Édition électronique

URL : http://journals.openedition.org/belgeo/11621

DOI : 10.4000/belgeo. 11621

ISSN : 2294-9135

Éditeur :

National Committee of Geography of Belgium, Société Royale Belge de Géographie

Édition imprimée

Date de publication : 1 janvier 2007

Pagination : 09/12/2013

ISSN : $1377-2368$

\section{Référence électronique}

Laurent Aujean, Etienne Castiau, Marcel Roelandts et Christian Vandermotten, « Le positionnement des villes belges dans le réseau global des services avancés », Belgeo [En ligne], 1 | 2007, mis en ligne le 07 août 2014, consulté le 01 mai 2019. URL : http://journals.openedition.org/belgeo/11621 ; DOI : $10.4000 /$ belgeo. 11621

Ce document a été généré automatiquement le 1 mai 2019.

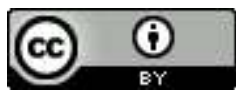

Belgeo est mis à disposition selon les termes de la licence Creative Commons Attribution 4.0 International. 


\title{
Le positionnement des villes belges dans le réseau global des services avancés
}

The positioning of Belgian cities in the global advanced services network

\author{
Laurent Aujean, Etienne Castiau, Marcel Roelandts et Christian \\ Vandermotten
}

\section{Introduction}

1 Les dynamiques de globalisation de l'économie qui se sont développées depuis les années 80 ont entraîné la domination de certaines villes dans les processus de commandement et de contrôle économiques. Ces villes devenues globales, parmi lesquelles figurent New York, Londres ou Tokyo, concentrent les sièges sociaux de multinationales de services avancés à la production ${ }^{1}$ qui caractérisent la nouvelle économie globale (Sassen, 1991). Les filiales de ces multinationales sont réparties à travers le monde selon un schéma qui suit et renforce une hiérarchie mondiale des villes (Friedman, 1986, 1995). En effet, les villes accueillant les ramifications de ces multinationales sont supposées être mises en relation par les flux d'informations et de connaissances entre les différents sièges d'une même compagnie, et s'insèrent dès lors dans un réseau de villes à l'échelle mondiale. Elles constituent les noeuds d'espaces de flux (Castells, 1996) aux connexions particulièrement complexes. Loin de construire une économie-monde plus ubiquiste, les nouvelles technologies de l'information, par la mondialisation des activités d'exécution qu'elles permettent, augmentent paradoxalement l'importance des noeuds principaux, y compris à travers les relations interpersonnelles de proximité qui peuvent s'y nouer.

2 A côté des grandes compagnies multinationales aux ramifications mondiales, se retrouvent des compagnies plus modestes, au rayonnement limité à une échelle continentale, nationale voire régionale. Il arrive assez fréquemment que ces firmes, davantage impliquées localement du fait d'affinités culturelles ou linguistiques, installant leurs filiales dans de plus petites villes, se retrouvent connectées au réseau des 
multinationales, soit pour bénéficier de flux de connaissances plus étendus et s'associer au nom d'un groupe mondialement reconnu, soit après rachat par ces multinationales. Un grand nombre de villes moyennes à petites peuvent dès lors se retrouver plus ou moins bien rattachées au réseau mondial des villes, et des connexions entre elles rendues possible dès lors que d'autres compagnies locales sont liées à une même multinationale.

3 Sur base de ces échanges supposés d'informations entre filiales, le Globalization and World Cities Group and Network (GaWC) de l'université de Loughborough (Taylor, 1997, 2001; Taylor et al., 2002) a développé une méthode de quantification des connexions inter-villes. La présence d'une filiale dans une ville permet en effet une quantification théorique des liens que cette ville entretient avec d'autres villes hébergeant des filiales de la même compagnie, en pondérant l'importance de chacune d'entre elles. Le lien sera considéré comme maximum si les deux villes abritent des sièges de haut rang décisionnel pour la même firme.

4 L'utilisation de cette méthodologie appelle toutefois deux remarques suffisamment importantes pour que les résultats soient utilisés avec prudence. La première concerne le présupposé d'un fonctionnement partiellement horizontal à l'intérieur des groupes, c'est à dire l'existence de liaisons fonctionnelles directes effectives entre filiales et bureaux régionaux, sans passer par les têtes des réseaux.

5 En effet, s'il va de soi que les flux verticaux (flux de directives, de connaissances et de comptes rendus) sont intenses entre les sièges de décision et leurs filiales, les transversalités dans les échanges d'information sont moins évidentes à établir, entre filiales d'un même niveau hiérarchique. Des échanges peuvent par exemple apparaître soit entre filiales complémentaires d'une même région, soit dans le suivi de dossiers d'une région à une autre du fait de déplacements de la clientèle, soit encore lors d'échanges dans le cadre de formations. Mais, dans de nombreux cas, il n'est pas du tout certain que les filiales communiquent entre elles de façon effective et régulière, en particulier lorsqu'elles se trouvent dans des régions économiquement concurrentes. La structure organisationnelle des firmes à établissements multiples reste souvent très hiérarchisée, ce que L. Halbert (2004), utilisant les flux téléphoniques des entreprises, a pu montrer dans sa thèse consacrée à la géographie des activités d'intermédiation en Région Ile-deFrance.

6 La deuxième remarque concerne la transposition d'une méthodologie appliquée jusqu'ici à une échelle mondiale (liens entre villes mondiales), à une échelle qui peut n'être que nationale dans le cas de firmes implantées uniquement dans les limites d'un seul pays. Dans l'analyse, le poids des filiales apparaît identique lorsqu'elles occupent un même niveau hiérarchique, qu'elles appartiennent à des multinationales ou soient des compagnies uniquement nationales, alors que les flux d'informations et de connaissances peuvent varier en intensité : les échanges d'informations peuvent gagner en importance lorsque la compagnie est nationale (affinités culturelles et linguistiques, distances physiques plus courtes...), mais par contre être davantage limités dans la transmission de nouvelles connaissances par rapport aux grosses multinationales développant intensivement l'innovation. Pour leur part, Cabus et Vanhaverbeke (2004) ont montré qu'à l'échelle de la Flandre, aucune preuve tangible ne pouvait être avancée concernant l'existence de réseaux inter-urbains basés sur d'intenses relations entre firmes.

7 Malgré ces réserves et en l'absence de données disponibles permettant de déterminer d'une manière plus précise les flux d'informations inter-villes ${ }^{2}$, l'étude présente a tenté de quantifier les relations des villes majeures belges, d'une part avec une série de villes 
internationales considérées comme des noeuds dans le réseau mondial des villes, d'autre part entre elles, en utilisant dans ses grandes lignes la méthodologie développée par le GaWC. Cette méthode a déjà été utilisée par Derudder et Taylor (2004) pour quantifier les connexions de Bruxelles et Anvers avec une série de villes mondiales, mais les autres villes belges n'avaient jusqu'à présent pas été intégrées dans une telle analyse ${ }^{3}$. Surtout, la quantification des connexions à une échelle nationale n'avait jamais été envisagée, les limites nationales apparaissant généralement comme des unités d'analyse négligeables dans "l'espace des flux transnationaux".

8 Les compagnies dont on a analysé la répartition des filiales appartiennent aux services avancés à la production et couvrent les secteurs de la banque et de la finance, de la comptabilité, des conseils juridiques, de la publicité, des conseils en ingénierie comprenant également les bureaux d'architectes, des conseils en management, dont les services informatiques, et des services logistiques.

9 L'article s'attache à établir le poids des villes belges, préalablement définies, dans les réseaux de firmes de services avancés à la production, selon la méthodologie développée par le GaWC, de façon à déterminer le degré de connectivité de ces villes aux réseaux des villes mondiales d'une part, entre elles d'autre part.

Il dresse également une cartographie du réseau national des villes belges, sur base de l'intensité des connexions inter-villes.

11 Le cas de Bruxelles sera traité de deux façons, d'une part dans sa dimension métropolitaine, d'autre part en scindant la Région de Bruxelles-Capitale et sa périphérie, située pour l'essentiel en Région flamande pour ce qui concerne la localisation des services avancés, de façon à évaluer le poids de cette dernière qui échappe à l'entité politique régionale.

\section{La connectivité des villes belges aux réseaux des villes mondiales et nationales}

\section{Choix des villes belges}

Les villes belges considérées comme les plus importantes ont été sélectionnées sur base de leur volume total d'emploi et de leur part d'emplois dans les secteurs des services avancés à la production. Seuls les emplois salariés ont été comptabilisés.

Les critères de sélection des villes se réfèrent à la méthodologie utilisée dans l'atlas GEMACA (1996) pour la détermination des pôles économiques, à savoir :

- soit une commune ayant un volume d'emploi salarié total supérieur à 35000 emplois, à laquelle s'ajoutent éventuellement les communes adjacentes présentant un volume d'emploi supérieur au même seuil ou une densité d'emploi supérieure à 700 par km² ;

- soit un ensemble de communes adjacentes ayant une densité d'emploi supérieure à 700 par $\mathrm{km}^{2}$ et qui, réunies, présentent un volume d'emploi total supérieur à 35000.

Parmi les ensembles urbains ainsi définis, n'ont été toutefois retenus que ceux présentant un volume d'emploi dans les services avancés à la production supérieur à 3000 et dont la part de cet emploi dans l'emploi total atteint au moins $5 \%$.

Les villes retenues (tableau 1 et figure 1) apparaissent ainsi au nombre de neuf, à savoir Bruxelles, Anvers, Gand, Liège, Charleroi, Hasselt-Genk, Bruges, Louvain et Malines ${ }^{4}$. Le 
noyau bruxellois inclut des communes adjacentes situées au nord-est, en Région flamande. Toutefois, afin de déterminer le poids de cette périphérie flamande, dans un contexte qui est souvent de concurrence inter-régionale, celle-ci a été individualisée en un sous-pôle urbain dénommé Zaventem, constitué des communes de Zaventem, Machelen et Vilvorde.

Tableau 1. Emploi salarié total et dans les services avancés dans les neuf villes majeures belges.

\begin{tabular}{lrcc}
\hline \multicolumn{1}{c}{ Villes majeures } & $\begin{array}{c}\text { Emploi } \\
\text { total }\end{array}$ & $\begin{array}{c}\text { Nombre d'emplois salariés } \\
\text { dans les services avancés }\end{array}$ & $\begin{array}{c}\text { Part de l'emploi salarié } \\
\text { dans les services } \\
\text { avancés (\%) }\end{array}$ \\
\hline Total Bruxelles & 690172 & 114817 & 17 \\
$\quad$ Région de Bruxelles-Capitale & 599193 & 98739 & 17 \\
Zaventem & 90979 & 16078 & 18 \\
Anvers & 272063 & 37498 & 14 \\
Gand & 134344 & 9965 & 7 \\
Liège & 94470 & 6424 & 6 \\
Hasselt-Genk & 84220 & 4757 & 5 \\
Charleroi & 79007 & 3836 & 13 \\
Louvain & 59591 & 7936 & 6 \\
Bruges & 57388 & 3178 & 9 \\
Malines & 35733 & 3322 & \\
\hline
\end{tabular}

\section{Source: ONSS 2001}

Figure 1. Les neuf villes majeures belges.

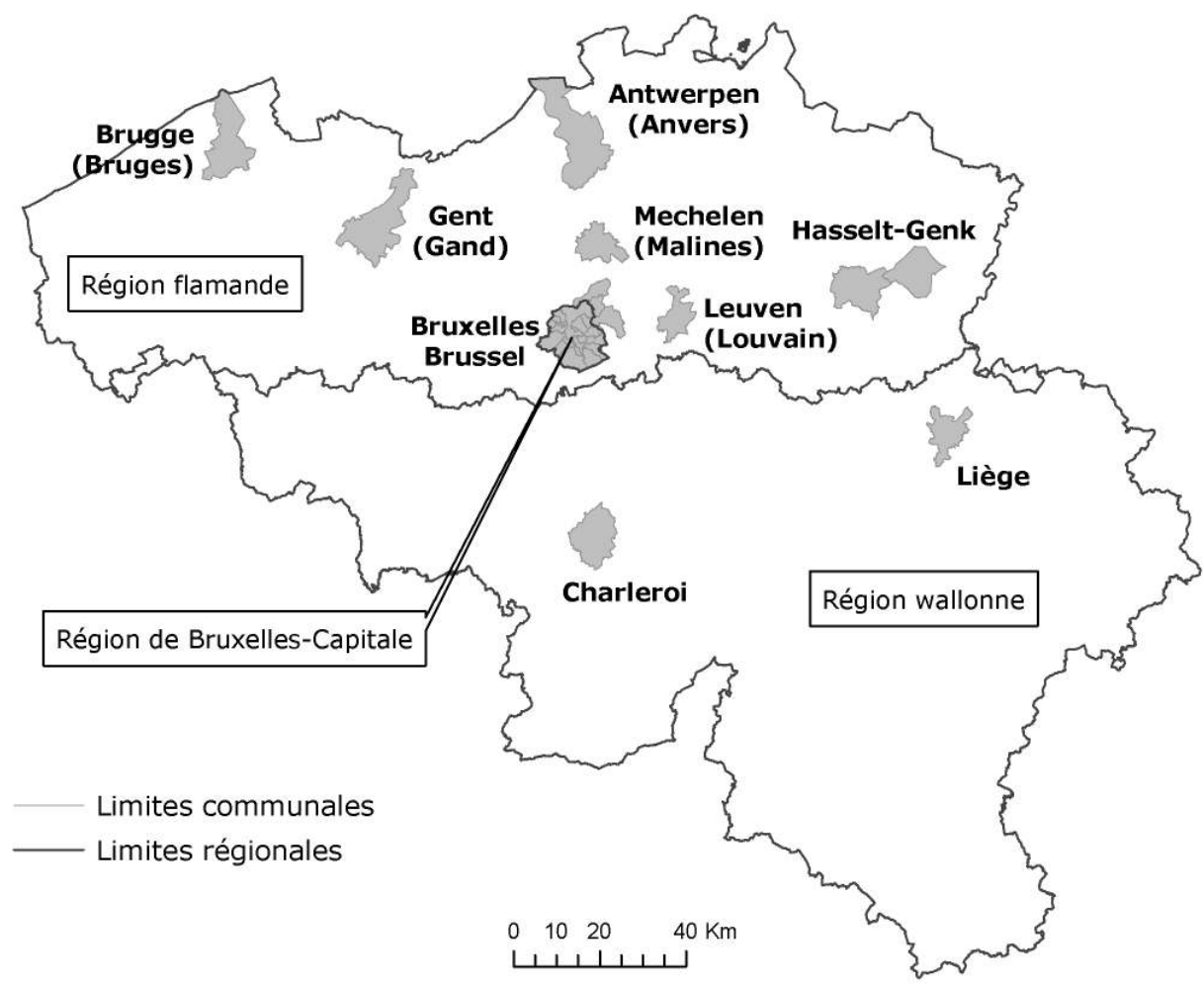




\section{Indices de connectivité des villes belges}

\section{Méthodologie}

La méthodologie utilisée s'appuie largement sur l'approche développée par le GaWC, permettant d'estimer le degré de connectivité des villes belges aux réseaux des villes mondiales et nationales, d'après leur poids dans les réseaux de firmes de services avancés à la production.

Une matrice $\mathrm{V}$ a été constituée en retenant d'une part 50 villes, c'est à dire les 9 villes belges plus 41 villes internationales ${ }^{5}$ considérées comme des noeuds majeurs dans le réseau mondial des villes, et d'autre part 323 firmes de services avancés, résultat d'une sélection parmi les plus importantes en Belgique ${ }^{6}$. Dans cette matrice $\mathrm{V}$, vij représente une valeur d'importance de la ville i pour la firme $\mathrm{j}$ qui $\mathrm{y}$ installe une filiale plus ou moins importante. Cette valeur varie de 1 à 3,1 dans le cas d'un bureau de très petite taille, voire un bureau de représentation, 2, qui représente la grande majorité des cotes attribuées, dans le cas d'une filiale classique ou d'une firme connectée à un réseau de firmes indépendantes, et enfin 3 lorsqu'il s'agit d'un siège social ou d'un important siège régional (par exemple, le siège européen d'une multinationale américaine). Une valeur nulle est attribuée en cas d'absence de filiale. Un exemple de matrice est présenté au tableau 2.

Tableau 2. Exemple de matrice, de 5 villes et 4 firmes.

\begin{tabular}{lcccc}
\hline \multicolumn{1}{c}{ Villes } & Firme A & Firme B & Firme C & Firme D \\
\hline New York & 3 & 0 & 3 & 2 \\
Hong Kong & 2 & 0 & 0 & 2 \\
Bruxelles & 2 & 3 & 2 & 2 \\
Gand & 2 & 2 & 0 & 1 \\
Liège & 2 & 2 & 0 & 0 \\
\hline
\end{tabular}

L'intensité de la connexion d'une ville belge bel avec une ville internationale int, pour une firme j, s'établit :

$\mathrm{r}_{\text {bel-int, } \mathrm{j}}={ }_{\mathrm{vbel}, \mathrm{j}} \cdot \mathrm{v}_{\mathrm{int}, \mathrm{j}}$

20 Ainsi dans l'exemple présenté, l'intensité de la connexion de Bruxelles avec New York, pour la firme A, est $2 \times 3=6$. Plus importantes seront les villes pour la firme $\mathrm{j}$ (valeur de 3 ), plus intenses seront les connexions entre ces deux villes. Lorsqu'on somme les intensités de connexion entre la ville belge bel et chacune des villes internationales, on obtient le degré de connectivité de cette ville au réseau international des villes, pour la firme $\mathrm{j}$ :

$\mathrm{r}_{\text {bel, }}=\Sigma \mathrm{r}_{\text {bel-int,j }}$

22 Dans l'exemple, le degré de connectivité de Bruxelles au réseau international des villes, pour la firme $\mathrm{A}$, est dès lors $6+4=10$. La somme des degrés de connectivité de la ville belge bel au réseau international des villes, pour chaque firme, permet d'obtenir l'indice de connectivité de cette ville au réseau international des villes:

ICint $_{\text {bel }}=\Sigma \mathrm{r}_{\text {bel, }}$

Bruxelles, dans l'exemple, obtient ainsi pour les 4 firmes considérées $10+0+6+8=24$. 

c'est-à-dire leur indice de connectivité internationale, Bruxelles et dans une moindre mesure Anvers dominent les autres villes. Elles apparaissent ainsi, en toute logique vu leur taille, comme les plus internationales des villes belges, concentrant de nombreuses firmes spécialisées dans les services avancés à la production.

Figure 2. Les indices de connectivité internationale des villes belges, avec et sans regroupement de la Région de Bruxelles-Capitale et Zaventem.

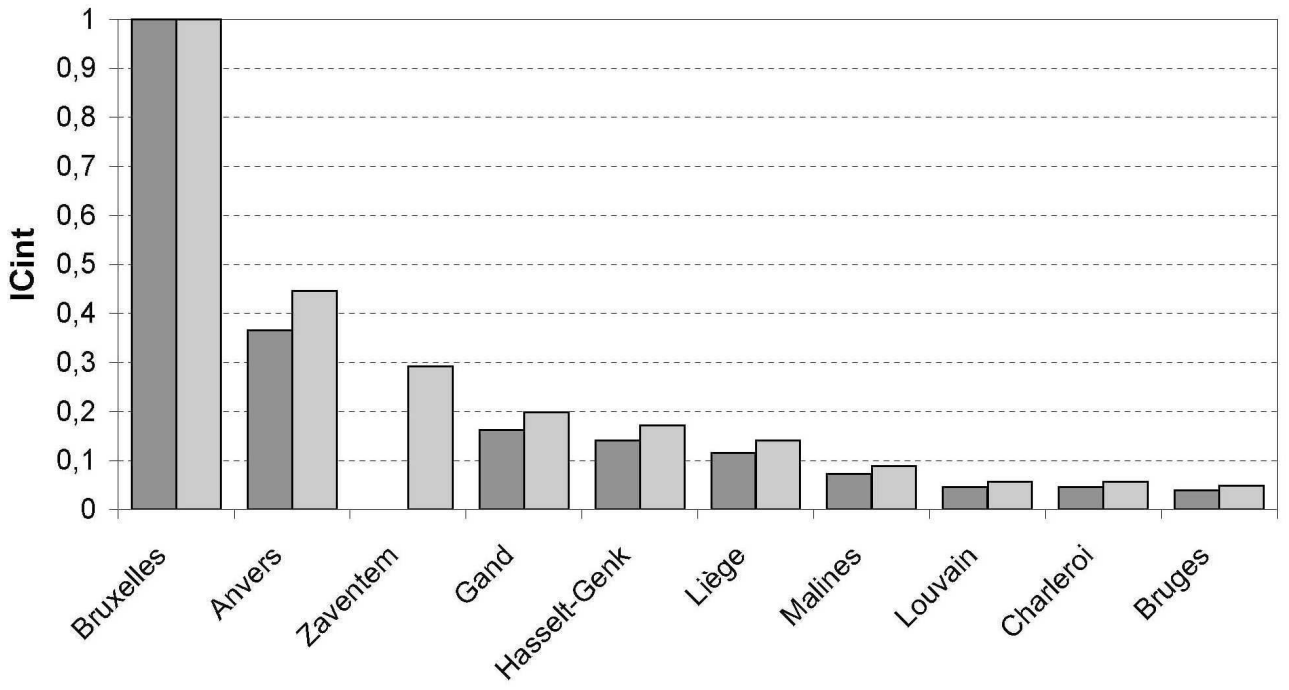

$\square$ Avec regroupement de la Région de Bruxelles-Capitale et Zaventem $\square$ Sans regroupement de la Région de Bruxelles-Capitale et Zaventem

L'écart de Bruxelles par rapport aux autres villes belges s'explique non seulement par sa position centrale sur le marché belge, tant francophone que flamand, et son rôle de capitale du capitalisme belge, mais sans doute plus encore par le fait que cette ville bénéficie de la présence d'organismes internationaux de premier plan, au premier rang desquels figurent les institutions européennes. Le principal aéroport national est implanté à Zaventem, commune flamande adjacente à la Région bruxelloise. Des universités et de nombreuses écoles supérieures, un recrutement qui touche pratiquement toutes les communes du pays lui assurent une importante main-d'oeuvre qualifiée. De nombreuses multinationales qui s'installent en Belgique choisissent de s'y implanter, sans chercher à installer de filiales dans les autres villes belges. Il s'agit en particulier de secteurs offrant des services très spécialisés, tels que la publicité ou les 
conseils juridiques spécialement liés à la présence d'organismes internationaux, dont la clientèle se trouve principalement concentrée dans la zone métropolitaine bruxelloise, voire très spécifiquement dans le quartier européen, ou de compagnies internationales actives dans la logistique aéroportuaire et basées autour de Zaventem.

Outre la présence de filiales "classiques" de nombreuses multinationales, la ville est parfois choisie comme siège européen et les firmes belges d'importance internationale y basent le plus souvent leur centre de décision.

Anvers bénéficie surtout de la présence de son port, deuxième européen après Rotterdam, mais plus spécialisé dans les marchandises générales plus productrices de valeur ajoutée, favorisant la concentration de services spécialisés dans les activités logistiques de transport maritime et d'entreposage portuaire. Cela apparaît sur la figure 3, présentant les ICint des villes belges pour chaque secteur d'activité composant les services avancés. L'importante activité industrielle de la région anversoise favorise par ailleurs la présence de services-conseil en ingénierie.

Figure 3. Les indices de connectivité internationale des villes belges pour chaque secteur des services avancés, avec scission entre la Région de Bruxelles-Capitale et Zaventem.
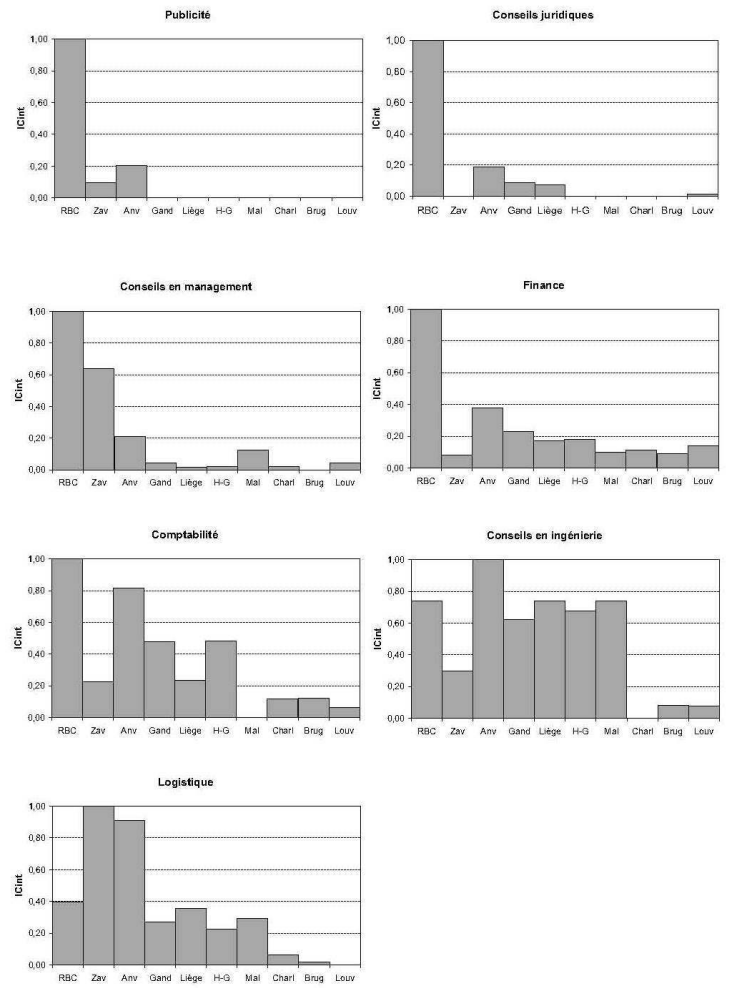

Quoique loin derrière Anvers, les villes de Gand, Hasselt-Genk et Liège ont une relative importance sur le plan international. Le port de Gand et son industrie, la situation d'Hasselt-Genk et de Liège sur des axes routiers et fluviaux importants, l'industrie automobile à Genk et la présence de l'aéroport de fret à Bierset, attirent quelques sociétés internationales de services logistiques et de conseils en ingénierie les raccordant au réseau international des villes.

Malines, et en particulier la portion de son territoire située en bordure de l'autoroute Bruxelles-Anvers, est choisie comme lieu d'implantation par quelques compagnies internationales. Sa proximité tant de Bruxelles que d'Anvers permet à ces firmes de 
couvrir les marchés de ces deux villes sans devoir installer une filiale dans chacune d'elles. $\mathrm{Vu}$ de l'étranger, ces firmes ne distinguent sans doute pas clairement Malines de Bruxelles.

Louvain, Charleroi et Bruges apparaissent de moindre intérêt pour les compagnies internationales. Louvain, malgré une forte proportion d'emplois dans les services avancés et suffisamment proche de Bruxelles pour être considérée comme insérée dans sa périphérie, peut toutefois paraître trop périphérique par rapport au coeur métropolitain représenté par l'axe Anvers-Bruxelles pour capter de nombreuses filiales de firmes internationales.

La mauvaise position de Charleroi, malgré la taille de la ville, s'explique surtout par la faiblesse historique de son secteur des services marchands, liée à une spécialisation dans l'industrie de base. Une situation d'héritage d'ancienne industrialisation lourde n'est pas propice au développement des services aux entreprises, en particulier avancés, comme en atteste la part de l'emploi dans ces secteurs, la plus faible parmi les villes belges considérées (tableau 1). La faible intégration des principales villes wallonnes dans les réseaux internationaux est d'ailleurs particulièrement marquante, à Liège y compris, pourtant première métropole wallonne.

Bruges enfin apparaît de faible importance dans le domaine des services avancés, tout au plus existe-t-il une certaine activité logistique d'importance internationale liée au port de Zeebrugge.

Lorsque Bruxelles est amputée de sa périphérie nord-est avec Zaventem, sa position reste tout aussi dominante, mais on constate toutefois la relative importance de cette périphérie sur le plan international, intermédiaire entre celle d'Anvers et celle de Gand (figure 2). L'aéroport de Zaventem, la proximité des axes autoroutiers, un moindre coût de l'immobilier et la disponibilité de terrains sont autant d'éléments attractifs pour des firmes consommatrices d'espace spécialisées dans les activités logistiques, qui évitent le centre de la zone métropolitaine, tel que cela apparaît à la figure 3. D'importantes compagnies internationales de conseils en management, dont notamment les services informatiques, et en ingénierie ont également préféré s'y installer plutôt qu'au centre, y bénéficiant d'une meilleure accessibilité et de coûts de l'immobilier plus avantageux.

L'analyse des relations inter-villes à l'échelle nationale (figure 4) montre certaines modifications dans le positionnement des villes belges. Bruxelles apparaît tellement orientée vers l'extérieur que l'importance de ses connectivités au niveau strictement national s'en trouve affaiblie, au point d'être talonnée par Anvers. Cette dernière, moins importante sur le plan international, est par contre souvent choisie par les compagnies de pratiquement tous les secteurs des services avancés comme second lieu d'implantation de filiale en Belgique, ce qui sur le plan national crée très souvent un lien entre Anvers et Bruxelles. Par ailleurs, Anvers accueille le siège principal de quelques firmes flamandes, voire de filiales de firmes néerlandaises ou allemandes, présentes également dans les autres villes flamandes et qui parfois s'implantent à proximité de Bruxelles, mais toutefois en dehors de ses limites telles que fixées par la méthodologie de l'analyse (Asse, Lot, Hoeilaart...), sous-estimant quelque peu les connectivités de la capitale au réseau des villes belges. Pratiquement tous les secteurs des services avancés sont fortement représentés à Anvers, comme le montre la figure 6, et tout particulièrement la logistique en relation avec son port. 
Figure 4. Les indices de connectivité nationale des villes belges, avec et sans regroupement de la Région de Bruxelles-Capitale et Zaventem.

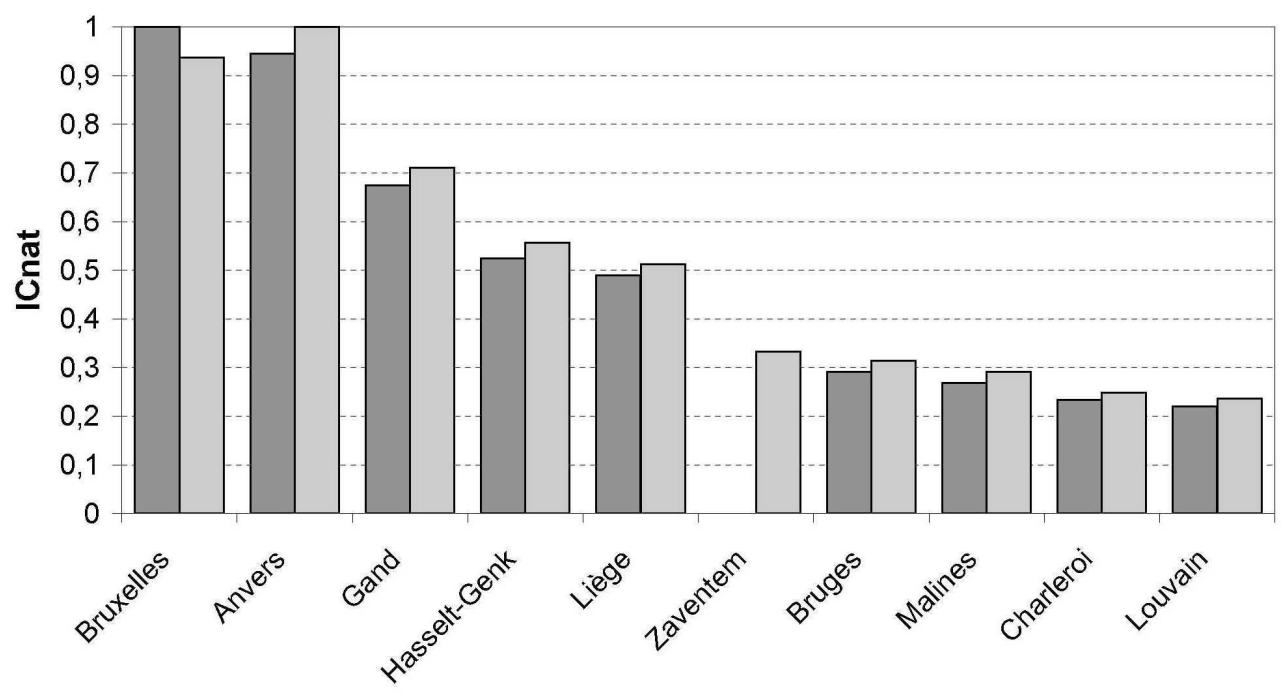

$\square$ Avec regroupement de la Région de Bruxelles-Capitale et Zaventem

$\square$ Sans regroupement de la Région de Bruxelles-Capitale et Zaventem

Lorsque Bruxelles est amputée de sa périphérie nord-est, la Région de Bruxelles-Capitale se voit même reléguée en seconde position derrière Anvers, par perte de firmes spécialisées notamment dans la logistique, l'ingénierie ou les conseils en management (figures 4 et 5).

Figure 5. Les indices de connectivité nationale des villes belges pour chaque secteur des services avancés, avec scission entre la Région de Bruxelles-Capitale et Zaventem.
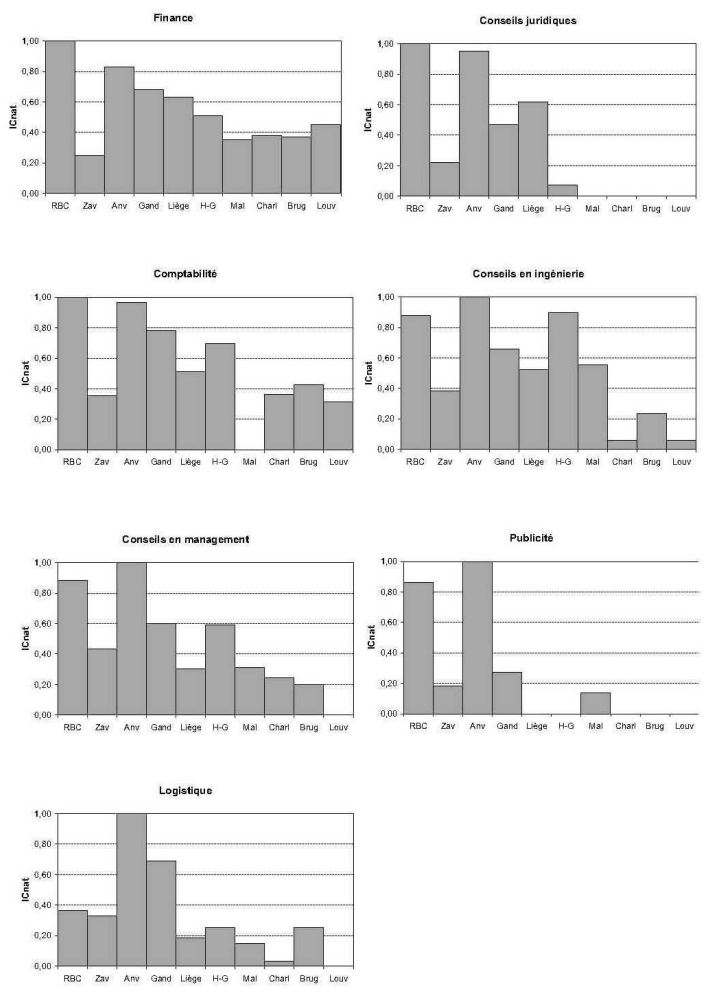
Gand démontre une connectivité relativement grande au réseau des villes belges, notamment grâce à son activité logistique qui la relie à Anvers en particulier. Elle devance largement Hasselt-Genk et Liège. Hasselt-Genk bénéficie particulièrement de la structure industrielle récente de sa région, notamment l'industrie automobile à Genk, qui favorise notamment l'implantation de firmes de conseil en ingénierie ou de services informatiques spécialisés dans les processus industriels.

Malines se retrouve cette fois parmi les villes les moins connectées au réseau national, endeçà de Bruges. Charleroi, faible en services avancés, est, comme précédemment, avantdernière, malgré sa taille et sa position de seconde ville wallonne.

Encore une fois, Liège, pourtant première métropole wallonne, se caractérise par son faible positionnement, loin derrière Gand et dépassée par Hasselt-Genk. La sousreprésentation des villes wallonnes dans les villes considérées peut l'expliquer, encore qu'elle traduise leur faiblesse objective en services avancés. Mais surtout, la faiblesse des relations de Liège avec les villes flamandes s'explique sans doute par un manque d'affinités linguistiques. Il en va de même pour Charleroi, où en outre la faiblesse de l'implantation des services avancés est encore plus patente. La faiblesse des liens entre Liège et Charleroi traduit aussi la faible intégration des deux grands bassins wallons et les héritages d'une industrialisation lourde peu différenciée. C'est principalement Bruxelles, bilingue, qui assure la connexion de Liège au réseau des villes belges, comme il apparaît sur la carte du réseau national des villes belges présentée en figure 6 .

Figure 6. Le réseau des villes belges.

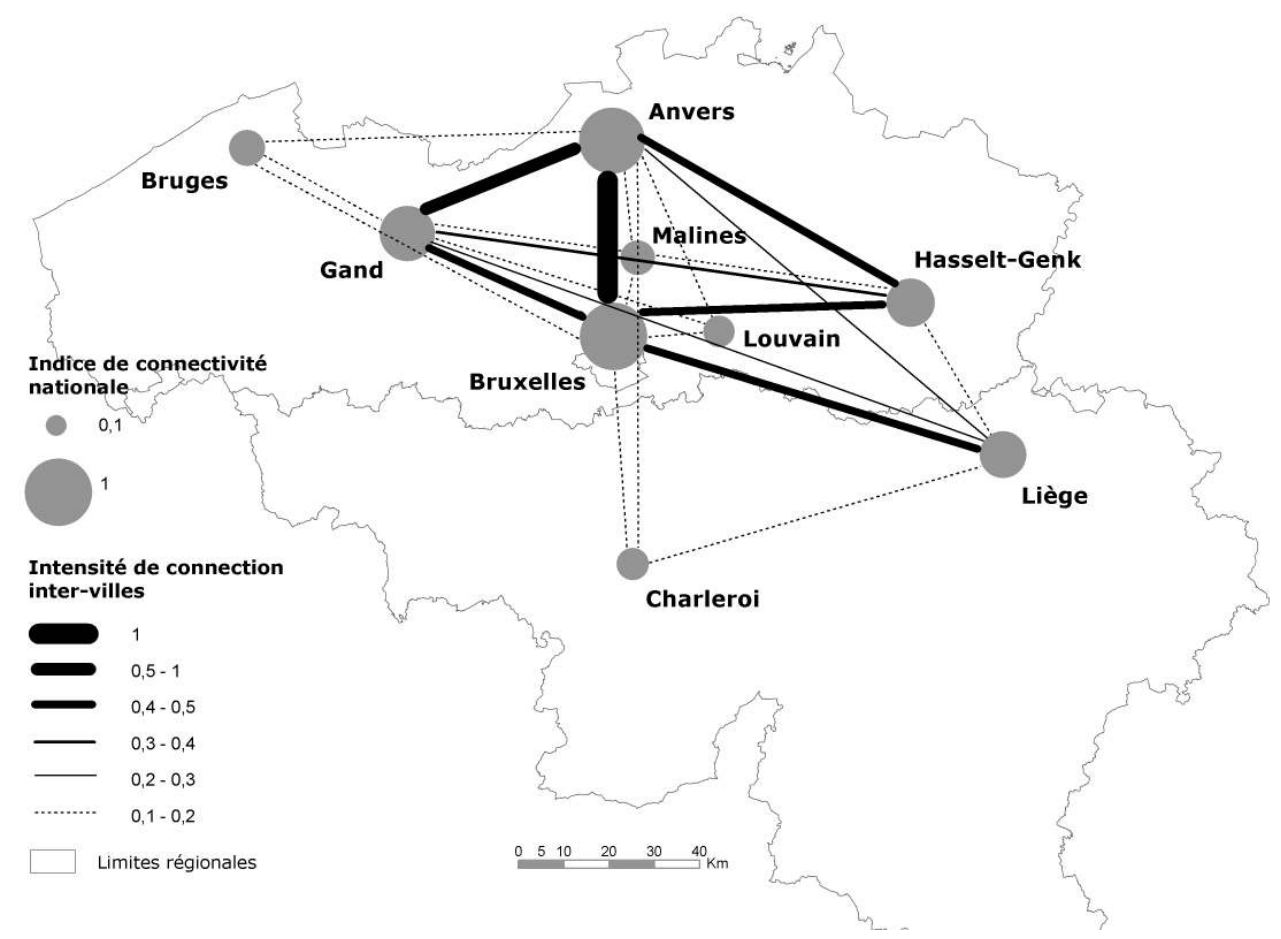

Celle-ci a été dressée sur base des intensités de connexion ${ }^{7}$ entre chaque paire de villes.

Bruxelles et Anvers, les deux plus grosses fournisseuses de main-d'oeuvre qualifiée, sont fréquemment choisies par les compagnies tant nationales qu'internationales, Bruxelles, bilingue, pour desservir le marché wallon et une partie du marché flamand, Anvers 
comme lieu d'implantation supplémentaire dans le marché flamand. On observe ainsi entre ces deux villes la plus forte intensité de relations.

Gand apparaît relativement bien connectée à Anvers, notamment parce qu'elle partage avec cette dernière des filiales et terminaux de compagnies actives dans les activités logistiques de transport maritime et de stockage portuaire. Sa liaison avec Bruxelles apparaît moindre, du même ordre que celles de Liège et d'Hasselt-Genk avec Bruxelles et de Hasselt-Genk avec Anvers. Gand, Hasselt-Genk et Liège représentent ainsi des villes de même niveau d'importance, desservant une clientèle plus locale.

Bruges se retrouve faiblement connectée, de même que Malines, siège de quelques multinationales profitant de la proximité tant de Bruxelles que d'Anvers.

Charleroi, désavantagé par sa faiblesse marquée dans les services avancés et Louvain, quelque peu excentré par rapport à l'axe Anvers-Bruxelles et en même temps périphérie éloignée de Bruxelles, se retrouvent isolées du réseau des villes belges.

Enfin, lorsque la Région de Bruxelles-Capitale et sa périphérie nord-est avec Zaventem sont scindées (figure 7), on remarque l'absence de liens significatifs entre elles. Elles sont de fait généralement considérées comme un seul et même ensemble métropolitain aux yeux des compagnies étrangères ou même nationales, qui installent très rarement une filiale dans chacune d'elles et choisissent plutôt une localisation dans l'une ou dans l'autre en fonction de la nature et de la consommation en espace de leur activité. Par contre, une connexion marquée s'observe avec Anvers, s'expliquant notamment par la présence de firmes de logistique et de conseils en ingénierie communes.

Figure 7. Le réseau des villes belges, avec scission entre la Région de Bruxelles-Capitale et Zaventem.

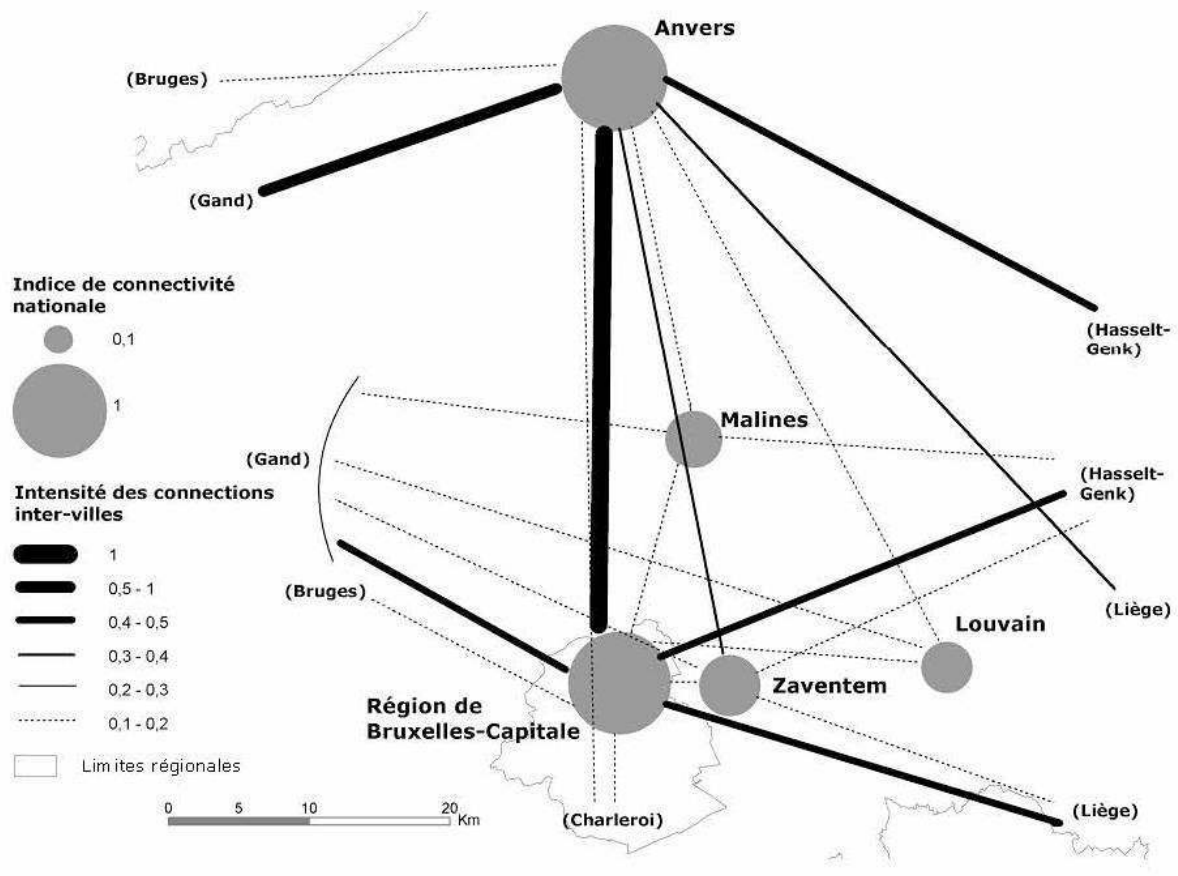




\section{Conclusions}

51 L'analyse du positionnement des villes belges dans les réseaux des villes internationales et nationales est basée sur une extension de la méthode développée par le GaWC. Elle a montré que Bruxelles est largement dominant sur le plan des relations entre villes impliquées dans les réseaux internationaux de firmes de services avancés à la production. Siège des institutions européennes et à la croisée des marchés francophones et flamands, Bruxelles est souvent choisie comme unique point d'ancrage en Belgique par de nombreuses multinationales. Cela rejoint les observations de Derudder et Taylor (2004), selon lesquels Bruxelles a des relations beaucoup plus globales qu'Anvers, dont l'importance est principalement européenne et les relations internationales plus limitées à des sous-secteurs spécifiques liés aux activités portuaires.

52 La dominance est au contraire bi-polaire, avec Bruxelles et Anvers, quand il s'agit des relations entre villes belges. Bruxelles perd en effet en importance et est talonnée par Anvers, généralement choisie comme second lieu d'implantation en Belgique de filiales de compagnies internationales ou nationales, parfois même de sièges principaux de firmes en rapport avec l'activité portuaire et industrielle de la région. Les relations d'Anvers avec la zone métropolitaine bruxelloise relèvent pour une part significative de liens avec la périphérie nord-est de celle-ci, qui connaît un important développement tertiaire, en particulier en logistique.

Les autres villes se positionnent loin derrière Bruxelles et Anvers. Gand occupe la troisième place, surtout marquée dans ses relations au niveau national, accueillant un certain nombre de firmes qui la lient tout particulièrement à Anvers (activités logistiques portuaires, design industriel). Liège, malgré son statut de première métropole wallonne, est dépassée par Hasselt-Genk, ce qui illustre la faiblesse de la Wallonie dans les secteurs des services typiquement liés à la globalisation de l'économie.

Les villes restantes apparaissent très peu connectées, notamment Charleroi, seconde ville wallonne mais pauvre en firmes dans les services avancés. Seule Malines joue un certain rôle sur le plan international en accueillant des firmes désireuses d'implanter leur filiale belge à proximité à la fois de Bruxelles et d'Anvers.

BIBLIOGRAPHIE

CASTELLS M. (1996), The Rise of the Network Society, Oxford, Blackwell.

CABUS P. and VANHAVERBEKE W. (2004), Ruimte en economie in Vlaanderen : Analyse en Beleidssuggesties, http://internet.vlaanderen.be/ ruimtevooreconomie/spre_publicaties_7.htm.

DERUDDER B. and TAYLOR P.J. (2003), “The global capacity of Belgium's major cities: Antwerp and Brussels compared", Belgeo, 4, pp. 459-476.

FRIEDMANN J. (1986), “The world city hypothesis”, Development and Change, 17, pp. 69-83. 
FRIEDMANN J. (1995), "Where we stand: a decade of world city research", in KNOX P.L., TAYLOR P.J. (eds.), World Cities in a World-System, CUP, Cambridge, pp. 21-47.

GEMACA (1996), Les régions métropoles de l'Europe du nord-ouest : limites géographiques et structures économiques, Studio IAURIF, Paris, $124 \mathrm{p}$.

HALBERT L. (2004), Densité, desserrement, polycentrisme et transformation économique des aires métropolitaines. Interpréter la concentration des activités d'intermédiation dans la zone centrale de la région francilienne, Thèse de doctorat, Laboratoire Dynamiques Sociales et Recomposition des Espaces, UFR de Géographie, Paris-I, Panthéon-Sorbonne.

HALL P. and PAIN K. (eds.) (2006), The Polycentric Metropolis: Learning from Mega-City Regions in Europe, London, Earthscan, 227 p.

SASSEN S. (1991), The Global City, Princeton University Press, Princeton (NJ).

TAYLOR P.J. (1997), "Hierarchical Tendencies amongst world cities: a global research proposal”, Cities, 14, 6, pp. 323-332.

TAYLOR P.J. (2001), "Specification of the World City network", Geographical Analysis, 33, pp. 181-194.

TAYLOR P.J. (2004), World City Network: A Global Urban Analysis, London, Routledge.

TAYLOR P.J., CATALANO G. and WALKER D.R.F. (2002), "Measurement of the world city network", Urban Studies, 39, 13, pp. 2367-2376.

\section{NOTES}

1. Il s'agit des services aux entreprises impliquant une main-d'oeuvre hautement qualifiée, des flux importants de connaissances, et ayant généralement connu des processus d'internationalisation. Il s'agit notamment des services financiers, des services de consultance, de la publicité et de la logistique.

2. L'étude de Cabus et Vanhaverbeke ne couvre que la Flandre et nous ne disposons pas d'autres sources.

3. L'étude présentée ici reprend en grande partie les résultats du projet POLYNET Interreg IIIB North-West Europe, consacré à l'étude du polycentrisme dans huit régions urbaines majeures d'Europe du nord-ouest, dont celle des "villes centrales belges" (Hall et Pain, 2006).

4. Ces villes correspondent à la plupart des métropoles et grandes villes belges définies au travers de leur aire urbaine fonctionnelle (FUA) dans le projet européen ESPON 1.4.3 - les grandes villes ont plus de 250000 habitants dans leur FUA, les métropoles plus de 500000 -, où toutefois Malines ne représente qu'une agglomération secondaire dans l'aire urbaine anversoise. MonsBorinage, portant les stigmates de son déclin industriel, correspond aux critères ESPON de grande ville (FUA de 274000 habitants) mais n'est pas représentée ici en raison de l'extrême faiblesse de son tertiaire lié aux services avancés à la production.

5. Ces villes, identifiées par le GaWC, sont Londres, Paris, Milan, Rome, Barcelone, Madrid, Amsterdam, Zurich, Francfort, Hambourg, Munich, Düsseldorf, Berlin, Copenhague, Stockholm, Vienne, Varsovie, Prague, Budapest, Moscou, Athènes, Istanboul, Lisbonne, Dublin, New York, Chicago, Los Angeles, San Francisco, Miami, Toronto, Mexico, Sao Paulo, Buenos Aires, Sydney, Melbourne, Mumbai, Djakarta, Singapour, Taipei, Hong Kong, Tokyo (Taylor, 2004)

6. Principalement sélectionnées selon le chiffre d'affaire dans le Trends Top 100 000, parmi les 100 premières firmes dans chaque sous-secteur des services avancés, en retenant celles qui soit ont une implantation dans au moins 2 villes belges, soit dans une ville belge et au moins une ville 
du réseau GaWC; en outre, ont été ajoutées plusieurs firmes plus modestes mais insérées dans des réseaux de filiales parfois étendus (exemple des bureaux de consultants juridiques), sélectionnées dans l'annuaire Europages et parmi celles utilisées dans les analyses du GaWC.

7. Etablies pour l'ensemble des firmes de services avancés présentes et rapportées à la plus forte valeur observée, c'est-à-dire Bruxelles-Anvers, de façon à varier entre 0 et 1.

\section{RÉSUMÉS}

Reprenant les concepts et méthodes issus des travaux sur les réseaux de villes globales, cet article a pour but de présenter une hiérarchie urbaine belge sous un nouveau regard, issu de l'analyse de la présence de firmes de services avancés à la production.

Après avoir sélectionné une dizaine de centres urbains majeurs, en apportant une attention particulière au cas de la Région de Bruxelles-Capitale et de sa très dynamique périphérie flamande autour de Zaventem, les niveaux de connexions de chaque ville ont été évalués, tant à l'échelle nationale qu'internationale.

Mise à part la domination de Bruxelles (et Anvers dans une moindre mesure), les positions de certaines villes belges apparaissent étonnantes. Par ailleurs, la hiérarchie se modifie selon l'échelle de travail (connectivités nationales ou internationales) et selon les secteurs. Ceci aide à comprendre les rapports des villes belges entre elles ainsi qu'au niveau international et la division géographique du travail qui se met en place dans la distribution des services avancés.

Starting from the concepts and methods developed by research on global cities networks, this article aims at presenting a new Belgian urban hierarchy, based on the analysis of the presence of advanced production services firms.

About ten major urban centres have been selected (with particular attention to the BrusselsCapital Region and its highly dynamic Flemish periphery around Zaventem) and their connection level assessed from a national and international point of view.

Aside from Brussels' (and to a lesser extent Antwerp's) predominance, the positions of some Belgian cities appear amazing. Moreover, the hierarchy changes according to level (national or international connectivity) and sector. This helps to understand Belgian cities' connections, both at national and international level, as well as the new geographical division of labour in the distribution of advanced services.

\section{INDEX}

Keywords : Belgium, cities network, advanced production services, cities, globalization

Mots-clés : Belgique, réseau de villes, services avancés à la production, villes, globalisation

\section{AUTEURS}

\section{LAURENT AUJEAN}

Institut de Gestion de l'Environnement et d'Aménagement du Territoire (IGEAT), Université Libre de Bruxelles 


\section{ETIENNE CASTIAU}

Institut de Gestion de l'Environnement et d'Aménagement du Territoire (IGEAT), Université Libre de Bruxelles, ecastiau@ulb.ac.be

\section{MARCEL ROELANDTS}

Institut de Gestion de l'Environnement et d'Aménagement du Territoire (IGEAT), Université Libre de Bruxelles, mroeland@ulb.ac.be

\section{CHRISTIAN VANDERMOTTEN}

Institut de Gestion de l'Environnement et d'Aménagement du Territoire (IGEAT), Université Libre de Bruxelles, cvdmotte@ulb.ac.be 